\title{
ON THE IDEAL STRUCTURE OF CERTAIN SEMIRINGS AND COMPACTIFICATION OF TOPOLOGICAL SPACES
}

\author{
BY \\ J. G. HORNE, JR.(1)
}

Introduction. Let $X$ be a topological space, $R(X)$ its family of real-valued continuous functions, and $\mathbb{S}(X)$ its family of open subsets. When it comes to reflecting the topological properties of $X$, there are many similarities between the ring and the lattice on $R(X)$, and a certain "strongly ordered" structure on $B(X)$. In this connection the works of Stone [17], Alexandrov [1], Kaplansky [10], Hewitt [8], Milgram [12], Shirota [15], and Henriksen [7] should be mentioned.

One similarity is that each has various families of "ideals" which admit intrinsically defined, compact topologies. A natural domain in which to study this situation is the semiring. It was recently called to our attention that one such study has already been made. Słowiskowski and Zawadowski studied the space of maximal ideals in "positive" semirings [16]. Our principal results concern the family of $R$-ideals in a class of semirings suggested by the $\mathbf{R}$-lattices of Shirota [15]. These semirings include various rings of continuous functions and the biregular rings (with identity) of Arens and Kaplansky [2], in addition to $R$-lattices. The notion of $R$-ideal is a generalization of the notions of lattice ideal and $O$-ideal of Milgram [12]. The present paper and [16] seem to overlap very little, except in some of the applications.

The author wishes to express his deep appreciation to the referee for his many helpful suggestions.

In $\S 0$, terms and conventions to be used throughout the work are given. The definitions of two particularly important relations, which are definable for any semigroup, appear in this section. These are the canonical order $O$, and (in the language of relations) its square, the strong canonical order.

$\$ 1$ contains the definition of $R$-ideal and $\breve{S i l o v}$ subset for any set $S$ with transitive order $R$. Included among examples of Šilov subsets are the Šilov semigroups of Civin and Yood [5]. Some elementary properties of $O$-ideals are proved in $\$ 2$. For an arbitrary relation $R$ on $S$, the notion of prime-like $(R)$ ideal is defined. This includes the familiar notion of prime ideal. The

Presented to the Society, November 19, 1955 under the title On semigroups and compactification theorems, December 28, 1955 under the title On o-ideals in $C(X)$, and December 21, 1956 under the title $o$-ideals in semirings and a universal compactification procedure; received by the editors January 23, 1957 and, in revised form, April 26, 1957 and May 23, 1957.

(1) A part of this work was supported by the National Science Foundation and is contained in the author's doctoral dissertation submitted to Tulane University. He wishes to thank Professor B. J. Pettis for his help and encouragement. 
most useful properties of maximal $R$-ideals, obtained in $\$ 4$, seem to derive from their being prime-like $(R)$.

The third section contains a general study of the Stone and dual Stone closure operations on an arbitrary family of subsets of an abstract set $S$. Criteria for compactness are obtained which seem particularly natural for application to families of subsets. An example shows that neither the maximal nor prime-like $O$-ideal space of a commutative ring with identity need be compact in the dual Stone topology.

We come to semirings in $\$ 4$. For the sake of completeness, we have included the definition of $R$-lattice. A definition of $R$-semiring at an element is given. It is for such semirings that we obtain some ideal structure theorems. The final section consists of applications to the study of $R$-ideals in the family of open subsets of a topological space $X$, the $O$-ideal structure of $R(X)$, and the ideal structure of biregular rings with identity.

0 . Preliminaries. The symbol $\subset$ denotes inclusion, while $<$ is used for proper inclusion. For sets $S$ and $A$ we write $S \backslash A=\{s \in S: s \notin A\}$. If $A$ is not empty and $A<S$, then $A$ is a proper subset of $S$. The empty set is denoted $\varnothing$.

A relation on $S$ is a subset of $S \times S$. If $R_{1}$ and $R_{2}$ are two relations on $S$ then $R_{1}$ is as strong as $R_{2}$ if $R_{1} \subset R_{2}$; that is, $a R_{1} b$ implies $a R_{2} b$. Those relations which are dense are of special interest here. $R$ is dense on $S$ if for $a, b \in S$ such that $a R b$, there is $c \in S$ satisfying $a R c$ and $c R b$. A relation $R$ directs a subset $A$ (or $A$ is $R$-directed) if for every pair $a$ and $a^{\prime} \in A$, there exists $e \in A$ such that $a R e$ and $a^{\prime} R e$. A frequently used relation on the family of subsets of a topological space $X$ is defined in terms of complete separation. Two subsets $A$ and $B$ of $X$ are said to be completely separated (written $A \mid B$ ) if there is a continuous real-valued function $f$ such that $f \mid A=0$ and $f \mid B=1$. For subsets $G$ and $H, G R H$ sometimes means $H \mid(X \backslash G)$.

If $S$ is endowed with a binary associative multiplication (indicated by juxtaposition or ".") then $S$ is a semigroup. For any subsets $A$ and $B$, the set $\{a b: a \in A$ and $b \in B\}$ is, as usual, denoted by $A B$. A nonempty subset $J$ is a left semigroup ideal (abbreviated 1.s.g. ideal) if $S J \subset J$. It is a semigroup ideal (abbreviated s.g. ideal) if $J S \subset J$ also. The center $C(S)$ of $S$ is the collection of elements $x \in S$ such that $x f=f x$ for all $f \in S$. An element $e \in S$ is a relative unit for $f \in S$ if $e \in C(S)$ and $f e=f$. An identity 1 and zero 0 satisfy $1 f=f 1=f$ and $0 f=f 0=0$, for all $f \in S$. Two relations on $S$ are especially important:

Definition 0.1. The canonical order on $S$ is the relation $O=\{(f, e): e$ is a relative unit for $f\}$.

Definition 0.2. The strong canonical order on $S$ is the relation $O^{2}$ $=\left\{(f, e):\right.$ for some $e^{\prime} \in C(S), f O e^{\prime}$ and $\left.e^{\prime} O e\right\}$.

If there is also a binary addition (written " + ") which is associative and commutative, and satisfies both $a(b+c)=a b+a c$ and $(b+c) a=b a+c a$, then $S$ is a semiring. $S$ is a semiring with identity or zero according as the semigroup $(S, \cdot)$ has an identity or zero. A left ideal (abbreviated lideal) $J$ in a 
semiring is a 1.s.g. ideal of $(S, \cdot)$ which also satisfies $J+J \subset J$. It is a (twosided) ideal if it is also a s.g. ideal of $(S, \cdot)$.

For a topological space $X$, we use $K(X)$ and $R(X)$ respectively to denote the rings of all continuous complex- and continuous real-valued functions on $X$. $K^{*}(X)$ and $R^{*}(X)$ denote the bounded members of $K(X)$ and $R(X)$. If $f \in K(X)$ then $Z(f)=\{x \in X: f(x)=0\}$ and $N(f)=X \backslash Z(f)$. If $A \subset X$ we use $A^{-}$and $A^{\circ}$ respectively for the closure and interior of $A$. If $X$ is locally compact and Hausdorff, $K_{\infty}(X)$ is the sub-ring of $K^{*}(X)$ of functions which vanish at infinity, and $K_{o}(X)$ is the sub-ring of $K_{\infty}(X)$ of functions with compact support: that is, those $f \in K_{\infty}(X)$ with $N(f)^{-}$compact.

1. $R$-ideals and Silov subsets. In this section, $S$ is a set with transitive relation $R$, no algebraic structure being assumed.

Definition 1.1. A subset $I$ of $S$ is an ideal with respect to $R$ if $f \in I, h \in S$ and $h R f$ imply $h \in I$.

The following definition is derived from Milgram's notion of $O$-ideal [12] and that of lattice ideal [3].

Definition 1.2. A nonempty subset $I$ of $S$ is an $R$-ideal if (i) $I$ is an ideal with respect to $R$, and (ii) $I$ is $R$-directed; that is, for $f, g \in I$ there is $e \in I$ such that $f R e$ and $g R e$.

An $R$-ideal $M$ is maximal if $M$ is a proper subset of $S$ and $M<M^{\prime}<S$ is false for every $R$-ideal $M^{\prime}$.

From some points of view certain subsets of $S$ can be much simpler than $S$ itself, and yet have essentially the same $R$-ideal structure (using the induced relation). Particularly easy to handle, yet of frequent occurrence, are the Silov subsets:

Definition 1.3. A subset $S_{o}$ of $S$ is a $\check{S}$ ilov $(R)$ subset (or simply a Šilov subset) provided the statements $f R e$ and $e R g$ for a triple $e, f, g \in S$ imply there is $e^{\prime} \in S_{o}$ such that $f R e^{\prime}$ and $e^{\prime} R g$. If $S$ is a semigroup and $S_{o}$ is a subsemigroup then $S_{o}$ is a Silov $(R)$ semigroup.

EXAMPLe 1.4. Let $X$ be a locally compact Hausdorff space and let $S$ be the multiplicative semigroup on $K_{o}(X)$. Suppose $R=O$, the canonical order on $S$. It is straightforward to prove that a subset $S_{o}$ of $S$ is a Silov subset if and only if whenever $F$ and $F^{\prime}$ are disjoint closed subsets of $X$ with $F$ compact, then there is $f^{\prime} \in S_{o}$ such that $f^{\prime} \mid F=1$ and $f^{\prime} \mid F^{\prime}=0$. A sub-semigroup having this property was called a Šilov semigroup by Civin and Yood [5], and was characterized algebraically in a way different from above.

EXAmple 1.5. Let $X$ be an arbitrary topological space and let $S$ be the multiplicative semigroup on $K(X)$. If $S_{o}$ is the collection of those functions taking their values in the closed unit interval, then $S_{o}$ is a Silov $(O)$ semigroup of $S$. For suppose $f e=f$ and $e g=e$. Let $\bar{e}$ denote the complex conjugate of $e$, and set $e^{\prime}=(e \bar{e}) \wedge 1$. Then $f e^{\prime}=f$ and $e^{\prime} g=e^{\prime}$. Since any semigroup in $S$ which is larger than a Šilov semigroup is also a Šilov semigroup, other examples are easy to obtain. In particular, $K^{*}(X)$ and $R^{*}(X)$ are Šilov semigroups of $K(X)$ and $R(X)$, respectively. 
EXAmple 1.6. Let $X$ be a topological space. If $G R H$ means either (i) $G \supset H^{-}$or (ii) $H \mid(X \backslash G)$ then the regular open sets form a Silov $(R)$ subset of the family of all open sets. For in either case, if $F R E$ and $E R G$ then $F R\left(E^{-}\right)^{\circ}$ and $\left(E^{-}\right)^{\circ} R G$. Thus it is apparent that Silov subsets of a semigroup need not be sub-semigroups.

In the following theorem we do not distinguish between a relation on $S$ and its restriction to a subset of $S$.

Theorem 1.7. Let $S_{o}$ be a Silov $(R)$ subset of $S$. Then these hold:

(1) If $I$ is an $R$-ideal in $S$ then $I \cap S_{o}$ is an $R$-ideal in $S_{o}$.

(2) If $I_{o}$ is an $R$-ideal in $S_{o}$ then $I \equiv L\left(I_{o}\right) \equiv\left\{f \in S: f R e\right.$ for some $\left.e \in I_{o}\right\}$ is an $R$-ideal in $S$ such that $I_{o}=I \cap S_{0}$.

(3) If $I_{1}$ and $I_{2}$ are distinct $R$-ideals in $S$ then $I_{1} \cap S_{o}$ and $I_{2} \cap S_{o}$ are distinct $R$-ideals in $S_{o}$.

Proof. Suppose $I$ is an $R$-ideal in $S$ and take $f_{1}, f_{2} \in I \cap S_{o}$. Let $e_{1}, e_{2}, e_{3} \in I$ be elements such that $f_{i} R e_{1}$ for $i=1,2$ and $e_{1} R e_{2} R e_{3}$. Then there is $g_{2} \in S_{o}$ such that $e_{1} R g_{2} R e_{3}$. Since $e_{3} \in I$, we have $g_{2} \in I \cap S_{o}$, and since $R$ is transitive $f_{i} R g_{2}$ for $i=1,2$. Therefore $I \cap S_{0}$ is $R$-directed. Since there exists $h \in I$, this argument also shows that $I \cap S_{0} \neq \varnothing$. Now if $f \in I \cap S_{0}$ and $h R f$ for some $h \in S_{o}$, then $h \in I$; hence $I \cap S_{o}$ is also an ideal with respect to $R$. It is therefore an $R$-ideal in $S_{o}$.

If $I_{o}$ is an $R$-ideal in $S_{o}$ then $I_{o} \subset L\left(I_{o}\right)$ so that $L\left(I_{o}\right)$ is $R$-directed. Since $R$ is transitive $L\left(I_{o}\right)$ is an ideal with respect to $R$. To see that $L\left(I_{o}\right) \cap S_{o}=I_{o}$, take $f \in L\left(I_{o}\right) \cap S_{o}$. There is $e_{o} \in I_{o}$ such that $f R e_{o}$. Since $I_{o}$ is an $R$-ideal in $S_{o}$, we have $f \in I_{\text {o }}$.

Finally, suppose $I_{1}$ and $I_{2}$ are $R$-ideals in $S$ with $g_{1} \in I_{1} \backslash I_{2}$. Take $e_{1}, e_{2} \in I_{1}$ so that $g_{1} R e_{1} R e_{2}$. Then neither $e_{1}$ nor $e_{2}$ is in $I_{2}$. Since $S_{o}$ is a Silov subset there is $e^{\prime \prime} \in S_{o}$ such that $g_{1} R e^{\prime \prime} R e_{2}$. The right hand relation implies $e^{\prime \prime} \in I_{1}$, and hence $e^{\prime \prime} \in I_{1} \cap S_{0}$. The first relation places $e^{\prime \prime}$ in $S \backslash I_{2}$.

For a topological space $X$, we have the following corollary, in virtue of Example 1.5.

CoRollary 1.8. The correspondence $I \rightarrow I \cap K^{*}(X)$ is one-to-one from the $O$-ideals in $K(X)$ onto the O-ideals in $K^{*}(X)$. If $M$ is a maximal $O$-ideal in $K(X)$ then $M \cap K^{*}(X)$ is a maximal O-ideal in $K^{*}(X)$.

The following result implies that the correspondence $I \rightarrow I \cap S_{o}$ induces a homeomorphism between $R$-ideals of $S$ and those of $S_{o}$, if these families of $R$-ideals are assigned the dual Stone paratopology, and $S_{o}$ is a Silov subset of $S$. (For a discussion of the dual Stone paratopology see $\$ 3$.)

Theorem 1.9. Let $M$ be an $R$-ideal of $S$ and $\mathfrak{A}$ a family of $R$-ideals of $S$. Then $M \subset \cup\{I: I \in \mathfrak{A}\}$ if and only if $M \cap S_{o} \subset \cup\left\{I \cap S_{o}: I \in \mathfrak{A}\right\}$.

Proof. Obviously, if $M \subset \cup\{I: I \in \mathfrak{A}\}$ then $M \cap S_{o} \subset \cup\left\{I \cap S_{o}: I \in \mathfrak{A}\right\}$. On the other hand, if $M \cap S_{o} \subset \cup\left\{I \cap S_{o}: I \in \mathfrak{A}\right\}$, and $f \in M$ is arbitrary, then 
elements $e, g \in M$ exist such that $f R e$ and $e R g$. Since $S_{o}$ is a Silov subset there is $e^{\prime} \in S_{o}$ such that $f R e^{\prime}$ and $e^{\prime} R g$. The second relation implies $e^{\prime} \in M \cap S_{o}$, so that for some $I \in \mathfrak{A}$, we have $e^{\prime} \in I \cap S_{o}$. From the first relation, $f \in I$ follows.

2. $O$-ideals and prime-like $(R)$ ideals. Now assume that $S$ is a semigroup. Recall that $f O e$ means: $f e=f$ and $e$ is in the center $C(S)$ of $S$. Hence an $O$-ideal is actually a (two-sided) s.g. ideal. In fact, we have the characterization:

(4) A subset $I$ of $S$ is an $O$-ideal if and only if $I$ is an l. (or r.) s.g. ideal such that for every pair $f_{1}, f_{2} \in I$ there is $e \in I$ such that $f_{i} O e$ for $i=1,2$.

Lemma 2.1. If $I_{1}$ and $I_{2}$ are $O$-ideals then $I_{1} \cap I_{2}$ and $I_{1} I_{2}$ are $O$-ideals, and $I_{1} \cap I_{2}=I_{1} I_{2}$.

Proof. We have $I_{1} I_{2} \subset I_{1} \cap I_{2}$ for any pair of s.g. ideals. Take $f \in I_{1} \cap I_{2}$; then $f \in I_{1} I_{2}$ holds since there is $e \in I_{2}$ such that $f=f e$.

To see that $I_{1} I_{2}$ is an $O$-ideal, suppose $h_{i}=f_{i} g_{i}$ with $f_{i} \in I_{1}$ and $g_{i} \in I_{2}$ for $i=1,2$. There are elements $e_{1} \in I_{1}$ and $e_{2} \in I_{2}$ such that $f_{i} O e_{1}$ and $g_{i} O e_{2}$ for $i=1,2$. Hence $I_{1} I_{2}$ contains the relative unit $e_{1} e_{2}$ for $h_{1}$ and $h_{2}$. Since $I_{1} I_{2}$ is also a s.g. ideal, it is an $O$-ideal by (4).

The following lemma implies that the family of $O$-ideals admits the dual Stone topology (see Theorem 3.10).

Lemma 2.2. If $I$ is an $O$-ideal and $J_{1}$ and $J_{2}$ are s.g. ideals and $I \subset J_{1} \cup J_{2}$ then $I \subset J_{1}$ or $I \subset J_{2}$.

Proof. Take $f \in I$ arbitrarily and suppose $I \nsubseteq J_{2}$. There are elements $f^{\prime}$ and $e \in I$ such that $f^{\prime} \notin J_{2}, f^{\prime} e=f^{\prime}$ (therefore $e \notin J_{2}$ ) and $f e=f$. Since $I \subset J_{1} \cup J_{2}$ we have $e \in J_{1}$. Therefore $f \in J_{1}$. Since $f \in I$ is arbitrary, we have $I \subset J_{1}$.

The notion of prime ideal has proved useful in many circumstances. However the notion of prime $O$-ideal is in general not fruitful. In many semigroups having an abundance of maximal $O$-ideals there are no proper prime $O$-ideals (see Remark 5.8). In many of these semigroups the maximal $O$-ideals may nonetheless be characterized as those $O$-ideals having a certain primelike property which we now define. $R$ is an arbitrary relation on $S$.

Definition 2.3. A subset $P$ of $S$ is prime-like $(R)$ provided $(S \backslash P)(U(S \backslash P)) \subset S \backslash P$, where $U(S \backslash P)=\{e \in S: f R e$ for some $f \in S \backslash P\}$. We refer to an $R$-ideal which is also a prime-like $(R)$ set as a prime-like $R$-ideal. A prime-like $(O)$ set is called simply a prime-like set.

An alternate form for the definition of "prime-like $(R)$ set," and the form most frequently used, is

(5) $P$ is a prime-like $(R)$ set if and only if whenever $f, e, k$ are elements in $S$ satisfying $f \notin P, f R e$ and $k e \in P$, then $k \in P$.

The following definition reduces, when $S$ is commutative and $P$ is an ideal, to the ordinary notion of prime-ness. 
Definition 2.4. A subset $P$ of $S$ is a prime set provided its complement $S \backslash P$ is a sub-semigroup; that is, $(S \backslash P)(S \backslash P) \subset S \backslash P$. A prime s.g. ideal or prime $O$-ideal is a prime set which is also a s.g. ideal or $O$-ideal, etc.

Some connections between these two notions are given in the following comments.

Remark 2.5. Prime sets are exactly the prime-like $(E)$ sets, where $E$ is the identity relation.

REMARK 2.6. A prime 1.(r.) s.g. ideal is a prime-like $(O)$ set, since in this case $U(S \backslash P) \subset(S \backslash P)$.

REMARK 2.7. Suppose a commutative semigroup $S$ has the property of von Neumann's regular rings [13]: $a \in S$ implies there is $x \in S$ such that $a x a=a$. Then every prime-like $(O)$ ideal $P$ is a prime ideal and the two notions coincide. For let $f$ and $g \in S \backslash P$, and suppose $g=g x g$. If $f g \in P$, then $f x g \in P$. However, $g O(x g)$ and $g \notin P$, so $f \in P$ by (5). This is a contradiction, so $f g \in P$ and $P$ is prime.

REMARK 2.8. A prime-like $(O)$ ideal may be neither a prime ideal, nor an $O$-ideal. Let $S$ be the multiplicative semigroup of continuous complex-valued functions on the closed unit interval. Choose $0<x<1$; then the set

$$
\{f \in S: Z(f) \supset(x, x+\epsilon) \text { for some } \epsilon>0\}
$$

has the desired properties.

We conclude this section with a list of examples.

Example 2.9. Let $S$ be the semigroup $(L, \wedge)$ of a lattice $(L, \vee, \wedge)$. Then $I$ is an $O$-ideal in $S$ if and only if $I$ is a (lattice) ideal [3], and is prime-like if and only if it is prime (see Remark 2.7 above).

Example 2.10 (Milgram, [12]). Let $X$ be a compact Hausdorff space. For each closed set $F \subset X$, the set $I(F)=\left\{f \in K(X): Z(f)^{\circ} \supset F\right\}$ is an $O$-ideal in the multiplicative semigroup on $K(X)$; and for every $O$-ideal $J$ of $K(X)$ there is a closed set $F \subset X$ such that $J=I(F)$. Note that the members of $I(F)$ consist exclusively of divisors of zero if and only if $I$ is proper, and in turn, if and only if $F$ is non-null.

EXAMPLE 2.11. Let $S$ be the family of open subsets of a topological space $S$. Define $G R H$ to mean $H \mid(X \backslash G)$. Then the maximal $R$-ideals are exactly the completely regular ends of P.S. Alexandrov [1]. Recall that a completely regular end is a family $\&$ of open sets which is maximal with respect to having the finite intersection property and being regular: for $G \in(S)$ there is $H \in(S)$ with $G R H$. The maximality implies that (B) is closed under finite intersection and hence is $R$-directed. Since a proper $R$-ideal is a regular family, the coincidence of the two classes follows.

EXAMPLE 2.12. Let $S$ be the multiplicative semigroup of a biregular ring with identity. Let $I$ be a (two-sided) ideal of $S$. For each $f_{1}$ and $f_{2} \in I$ there are (idempotent) elements $e_{1}$ and $e_{2} \in C(S) \cap I$ such that $f_{i} e_{i}=f_{i}$, for $i=1,2$. Since $e_{1}$ and $e_{2}$ commute with each other, $e_{1}+e_{2}-e_{1} e_{2}$ is a relative unit for both $f_{1}$ and $f_{2}$. Thus every two-sided ideal of $S$ is an $O$-ideal. 
Example 2.13. Let $L$ be an $R$-lattice with respect to a relation $R$. An open ideal was defined in [15] to be a subset $I$ of $L$ satisfying: (1) $0 \notin I$, (2) $f$ and $g \in I$ imply $f \wedge g \in I$, and (3) for each $f \in I$ there is $g \in I$ such that $f R \mathrm{~g}$. The set $I$ is a maximal open ideal if it is maximal with respect to (1)-(3). We shall sketch an argument to show that the maximal open ideals of $L$ are precisely the maximal $R$-ideals of $L$. The missing details involve the definition of $R$-lattice, which appears as our Definition 4.1 , or those consequences of it which are discussed immediately after 4.1 . Briefly, the procedure is to show: (a) every maximal open ideal is a proper $R$-ideal, (b) every proper $R$-ideal is an open ideal, and (c) every open ideal is contained in a maximal open ideal. To show (a), first note that every set satisfying (2) and (3) is $R$-directed. Next, if $I$ is an open ideal, then $L(I)=\{f \in L: f R e$ for some $e \in I\}$ is an open ideal, which, by (3), contains $I$. Therefore, if $I$ is a maximal open ideal, then $L(I)=I$, so $I$ is an ideal with respect to $R$. Hence every maximal open ideal is a (proper) $R$-ideal. To show (b), first show that if $f \neq 0$, then $f R 0$, so that no proper $R$-ideal contains 0 . Now an $R$-ideal $I$ obviously satisfies (3); that it satisfies (2) comes from the fact that $I$ is $R$-directed, property (ii) of 4.1 , and the fact that $I$ is an ideal with respect to $R$. Finally, (c) is a consequence of Zorn's lemma.

EXAmple 2.14. Let $S$ be the multiplicative semigroup on $R(X)$ with $X$ a completely regular Hausdorff space. The sets $N^{p}$ of Gillman and Henriksen [6] are exactly the maximal $O$-ideals in $S$. This follows easily from Theorem 5.10 and the comments preceding it.

3. Topological preliminaries. Let $S$ be a set and $\subseteq$ some family of proper subsets of $S$. It is appropriate to refer to the operations, defined for subsets $\mathfrak{A}$ of $\subseteq$ by

(6) $c_{1}(\mathfrak{U})=\left\{M_{o} \in \mathfrak{S}: M_{o} \subset \cup\{M: M \in \mathfrak{A}\}\right\}$ and,

(7) $c_{2}(\mathfrak{A})=\left\{M_{o} \in \mathfrak{S}: M_{o} \supset \cap\{M: M \in \mathfrak{A}\}\right\}$

as the dual Stone and Stone closure operations respectively (see [17;9], and [4] for example). When the context makes confusion unlikely, we shall write $\mathfrak{A}-$ for $c_{1}(\mathfrak{H})$ as well as $c_{2}(\mathfrak{H})$. These operations need not be closure operations in a topology, but they can fail only because $c_{i}(\mathfrak{A} \cup \mathfrak{B}) \subset c_{i}(\mathfrak{A}) \cup c_{i}(\mathfrak{B})$ may fail for $i=1$ or 2 (see [4]). Nevertheless, if those sets $\mathfrak{A}$ such that $\mathfrak{A}=c_{i}(\mathfrak{A})$ are taken to be closed $\left(c_{i}\right)$ and their complements are called open $\left(c_{i}\right)$, the family of all open $\left(c_{i}\right)$ sets satisfies the condition for being a paratopology [11]. We speak of the family of all open $\left(c_{1}\right)$ sets as the dual Stone paratopology on $\subseteq$ and the family of all open $\left(c_{2}\right)$ sets as the Stone paratopology on $\subseteq$. We also say that a given operation determines its associated paratopology. An elementary fact used of ten without mention is that if $\mathfrak{S}_{1}$ and $\mathfrak{S}_{2}$ are two families of sets and $\mathfrak{S}_{1} \subset \Im_{2}$, then the dual Stone paratopology on $\mathfrak{S}_{1}$ is the same as the paratopology induced on $\mathfrak{S}_{1}$ by the dual Stone paratopology on $\Im_{2}$; a corresponding statement holds for the Stone paratopology.

Under the name topology, Nöebling [14] has shown that the usual definitions of continuity and compactness continue to have meaning for para- 
topological spaces, and many of the usual theorems remain valid. Of interest here are (i) the equivalence of the open sets and closed sets criteria for compactness and (ii) the theorem that the continuous image of a compact paratopology is compact.

Let $T$ be a paratopology and let $T^{\prime}$ be the collection of complements of members of $\mathfrak{T}$. A family $\mathfrak{S}$ of sets is a closed [open] base for $\mathfrak{T}$ if $\mathfrak{S} \subset \mathfrak{T}^{\prime}[\mathfrak{S} \subset \mathfrak{T}$ ] and if every member of $\mathfrak{T}^{\prime}[\mathfrak{T}$ ] is the intersection [union] of members of $\mathfrak{H}$. The members of a closed [open] base are referred to as basic closed [open] sets.

For $f \in S$ we define the families

(8) $\mathfrak{u}(f)=\{M \in \subseteq: f \in M\}$, and

(9) $\mathfrak{F}(f)=\{M \in \Im: f \notin M\}$.

Then the sets $\mathfrak{U}(f)[\mathfrak{F}(f)]$ form an open [closed] base for the dual Stone paratopology and they form a closed [open] base for the Stone paratopology.

In the first part of this section we prove several elementary, purely settheoretic, lemmas concerning compactness. These are more or less implicit in much work of this sort, but it seems that they have never been stated explicitly.

Evidently $\mathfrak{S}$ is compact if and only if every family of basic closed sets with the finite intersection property has a non-null intersection. Now a family $\{\mathfrak{F}(f): f \in A \subset S\}[\{\mathfrak{u}(f): f \in A \subset S\}]$ of basic closed $\left(c_{1}\right)\left[\left(c_{2}\right)\right]$ sets has non-null intersection if and only if there is an $M_{o} \in \mathfrak{S}$ such that

$$
M_{0} \subset S \backslash A\left[M_{0} \supset A\right],
$$

so the following is immediate:

THEOREM 3.1. The dual Stone [Stone] paratopology on $\mathbb{S}$ is compact if and only if for every set $A \subset S$ having the property

(10) [(11)] For any finite subset $F$ of $A$ there is $M \in \subseteq$ such that $M \subset S \backslash F[M \supset F]$, there is $M_{o} \in \subseteq$ such that $M_{o} \subset S \backslash A\left[M_{o} \supset A\right]$.

Definition 3.2. A set $A$ which satisfies condition (10) [(11)] of the theorem will be called an $f$-set $\left(c_{1}\right)\left[\left(c_{2}\right)\right]$ with respect to $\subseteq$ (or when no confusion can result, simply an $f$-set $)$.

Since satisfying condition (10) $[(11)]$ is a property of finite character, every $f$-set is contained in a maximal $f$-set. Proving compactness $\left(c_{1}\right)\left[\left(c_{2}\right)\right]$ is equivalent to proving that if $A$ is a maximal $f$-set then $S \backslash A[A]$ is in $\subseteq$. The following criterion of maximality of an $f$-set is useful. Its verification is quite standard, and is omitted.

Lemma 3.3. Anf-set $\left(c_{1}\right)\left[\left(c_{2}\right)\right] A$ is maximal if and only if it satisfies the condition

(12) $[(13)] h \notin A$ implies there is a finite set $F$ in $A$ so that $M \in \subseteq$ implies $M \cap(F \cup\{h\}) \neq \varnothing[M D(F \cup\{h\})]$. 
Definition 3.4. A subset $F$ of $S$ is covering $\left(c_{1}\right)\left[\left(c_{2}\right)\right]$ with respect to $\subseteq$ if $M \in \subseteq$ implies $M \cap F \neq \varnothing[M D F]$. When no confusion can result we say simply that $F$ is covering.

Theorem 3.5. Suppose $S$ is a set and $\subseteq$ is some family of proper subsets of $S$. Let $A$ be a maximal $f$-set $\left(c_{1}\right)\left[\left(c_{2}\right)\right]$ with respect to $\subseteq$. Then

(14) if for some relation $R$ on $S$, members of $\subseteq$ are ideals with respect to $R$ then $S \backslash A[A]$ is an ideal with respect to $R$;

(15) if $E$ is some $n$-fold cartesian product of $S$, if $\alpha: E \rightarrow S$ is a function such that for every $M \in \subseteq$, and $a_{1}, a_{2}, \cdots, a_{n} \in M, \alpha\left(a_{1}, a_{2}, \cdots, a_{n}\right) \in M$ then $a_{1}, a_{2}, \cdots, a_{n} \in S \backslash A[A]$ implies $\alpha\left(a_{1}, a_{2}, \cdots, a_{n}\right) \in S \backslash A[A]$;

(16) if $S$ is a semigroup, $R$ is a relation on $S$ and all members of $S$ are primelike $(R)$ sets then $S \backslash A[A]$ is a prime-like $(R)$ set.

Proof. Let $A$ be a maximal $f$-set $\left(c_{1}\right)$ and assume that members of $\subseteq$ are ideals with respect to $R$. Take $f \in S \backslash A$, and suppose $h \in S$ satisfies $h R f$. By Lemma 3.3 there is a finite set $F$ in $A$ such that $F \cup\{f\}$ is covering $\left(c_{1}\right)$. It follows that the set $F \cup\{h\}$ is covering. For take $M \in \subseteq$ and suppose $M \cap F=\varnothing$. Then $f \in M$ and therefore we have $h \in M$. Thus, since $F \cup\{h\}$ is a finite set such that $M \in \subseteq$ implies $M \cap(F \cup\{h\}) \neq \varnothing$, and $A$ is an $f$-set, we have $h \in S \backslash A$.

To see (15), take $a_{1}, a_{2}, \cdots, a_{n} \in S \backslash A$. Corresponding to each $a_{i}$, $i=1,2, \cdots, n$, there is a finite set $F_{i}$ in $A$ such that $F_{i} \cup\left\{a_{i}\right\}$ is a covering set. Now if $F=\cup_{i-1}^{n} F_{i}$ then the set $\left\{\alpha\left(a_{1}, a_{2}, \cdots, a_{n}\right)\right\} \cup F$ is a covering set. For if $M \in \subseteq$ and $M \cap F=\varnothing$ then we have $a_{i} \in M$ for each $i=1,2, \cdots, n$. Therefore $\alpha\left(a_{1}, a_{2}, \cdots, a_{n}\right) \in M$. Therefore $\alpha\left(a_{1}, a_{2}, \cdots, a_{n}\right)$ is in $S \backslash A$, since, as above, it is an element which forms a covering set when adjoined to a finite subset $F$ of $A$.

Suppose now that $A$ is a maximal $f$-set $\left(c_{2}\right)$ and that members of $S$ are prime-like $(R)$ sets. Take $f \notin A, e \in S$ such that $f R e$ and assume $k e$ is in $A$. There is a finite subset $F_{o}$ of $A$ such that $F_{o} \cup\{f\}$ is a covering set $\left(c_{2}\right)$. We show $k \in A$ by showing that for arbitrary finite $F$ in $A$ there is $M \in \Im$ such that $F \cup\{k\} \subset M$. For any such set $F$, there is $M \in \subseteq$ which contains $F \cup F_{o} \cup\{k e\}$. Therefore $f \notin M$. Since $M$ is prime-like $(R)$, we have $k \in M$, and hence $F \cup\{k\} \subset M$. It follows that $k$ is in $A$ and that $A$ is a prime-like $(R)$ set.

The remaining arguments are similar to those given and are omitted.

The following is a partial list of consequences of the theorem. It includes a well known result of Wallman for lattices [18] and of Stone for commutative rings with identity [17].

Corollary 3.6. Let $S$ be a semiring with zero [identity], and let $R$ be a relation on $S$. Then these families are compact in the dual Stone [Stone] paratopology: 
ideals in $S$.

$$
\text { the collection of all }\left\{\begin{array}{l}
\text { proper }[\text { proper }] \\
{[\text { maximal] }} \\
\text { proper prime-like }(R) \\
{[\text { proper prime-like }(R)]}
\end{array}\right\}\left\{\begin{array}{l}
\text { left } \\
\text { right } \\
\text { two-sided }
\end{array}\right\}
$$

Proof. We may as well suppose the given family of ideals is not empty. In every case, if $A$ is a maximal $f$-set $\left(c_{1}\right)\left[\left(c_{2}\right)\right]$ then $S \backslash A[A]$ is a proper subset $S$. For $S \backslash A \neq \varnothing[A \neq S]$ if $S$ has a zero [identity], and $S \backslash A \neq S[A \neq \varnothing]$ since the family of ideals concerned is not empty. In all cases except those concerning maximality, the set $S \backslash A[A]$ is in $\subseteq$ by the theorem. In case $\mathbb{S}$ consists of maximal elements, then $A$ is also maximal. For if $A<B$, with $B \in \mathfrak{S}$, then $B$ is a larger $f$-set $\left(c_{2}\right)$ than $A$, contrary to the maximality of $A$. Thus $A$ is in $\mathfrak{S}$ in these cases also. The compactness now follows from the remarks prior to Lemma 3.3.

REMARK 3.7. By Remark 2.5, this corollary implies that the collection of all prime left ideals, etc., in a semiring with zero [identity] is compact in the dual Stone [Stone] paratopology.

EXAmple 3.8. The collection $\mathfrak{M}(S)$ of maximal $O$-ideals in a commutative ring $S$ with identity need not be compact in the dual Stone paratopology. Let $X$ be the set of positive reals with the usual topology. Let $G R H$ mean that $H$ is completely separated from $X \backslash G$. Let $\omega$ denote the collection of positive integers, and for each $n \in \omega$ choose a sequence $\left\{G_{i n}\right\}_{i}$ of open intervals such that $G_{i n} R G_{i+1 n}, \bigcap_{i}\left\{G_{i n}\right\}=\{n\}$ and if $n \neq m$ then the closures of $G_{1 n}$ and $G_{1 m}$ are disjoint. For each $i$ and $n \in \omega$ let $e_{i n}$ be the continuous function such that $e_{i n}\left|\left(X \backslash G_{i n}\right)=1, e_{i n}\right| G_{i+1 n}=0$, and $e_{i n}$ is linear in the two intervals whose union is $G_{i n} \cap\left(X \backslash G_{i+1 n}\right)$. Let $S$ be the ring generated by the $e_{i n}$, the function $e_{00}$ that is identically 1 , and its negative $e_{01}$. The members of $S$ can be written in the form $e_{1}+e_{2}+\cdots+e_{n}$ where each $e_{i}$ is a finite product of the $e_{i n}$. It follows that $M$ is a maximal $O$-ideal if and only if for some $n \in \omega, M=M_{n}=\left\{f \in S: n \in Z(f)^{\circ}\right\}$. Thus for each $n \in \omega$, the open set $\mathfrak{U}\left(e_{1 n}\right)$ $=\left\{M_{n}\right\}$; so $\mathfrak{M}(S)$ is an infinite discrete space, and hence a noncompact space, in the dual Stone paratopology.

In this example, the paratopology is actually a topology (see Theorem 3.10). Further, the classes of maximal and prime-like $O$-ideals coincide. For the $M_{n}$ are obviously prime-like. And in any ring $S$ with 1 , if $M$ is a primelike $O$-ideal, then $M$ is maximal. For suppose $M<M^{\prime}$, where $M^{\prime}$ is an $O$-ideal. There exist $e_{1}, e_{2}$ and $f \in M^{\prime} \backslash M$ such that $f O e_{1} O e_{2}$. Then $\left(1-e_{2}\right) e_{1}=0 \in M$. Therefore $1-e_{2} \in M \subset M^{\prime}$, so that $1 \in M^{\prime}$. Hence $M^{\prime}=S$, and $M$ is maximal.

We have, however, these criteria for compactness $\left(c_{1}\right)$.

THEOREM 3.9. Let $S$ be a semigroup with zero and let $\subseteq$ be a non-null family of proper $R$-ideals in $S$. Assume that $f R g$ and $f R h$ imply $f R g h$, and $R$-ideals are s.g. ideals. Then each of the following conditions implies the one below it.

(18) $\subseteq$ is compact in the dual Stone paratopology. 
(19) The intersection of the members of a non-null closed family $\mathfrak{A} \subset \subseteq$ is an R-ideal.

(20) If $F$ is a finite subset of $S$ which is not covering, and $f, g \in S$ are elements such that the sets $\{f\} \cup F$ and $\{g\} \cup F$ are covering, then there is $e \in S$ such that $f R e, g R e$ and the set $\{e\} \cup F$ is covering.

(21) If $A$ is a maximal $f$-set $\left(c_{1}\right)$ then $S \backslash A$ is an $R$-ideal.

Hence if $\subseteq$ consists either of the collection of all proper $R$-ideals, prime $R$ $i d e a l s$, or prime-like $R$-ideals, then the conditions are equivalent.

Proof. Suppose $\mathfrak{S}$ is compact and let $\mathfrak{A}$ be a closed and non-null subset. Let $I(\mathfrak{A})=\bigcap\{M: M \in \mathfrak{A}\} . I(\mathfrak{H})$ is not empty (since every s.g. ideal contains zero), and it is obviously an ideal with respect to $R$. Now take $f, g \in I(\mathfrak{H})$. For each $M \in \mathfrak{A}$, there is $e \in M$ such that $f R e$ and $g R e$. That is, the collection $\{\mathfrak{U}(e): e \in M$ for some $M \in \mathfrak{A}$ and $f R e, g R e\}$ is an open cover of the compact set $\mathfrak{A}$. Therefore there is a finite set $e_{1}, e_{2}, \cdots, e_{n} \in S$ such that every $M \in \mathfrak{A}$ contains some $e_{i}$, and such that $f R e_{i}$ and $g R e_{i}$ for each $i=1,2, \cdots, n$. Take $e_{o}=\prod_{i=1}^{n} e_{i}$. By hypothesis we have $f R e_{o}$ and $g R e_{o}$. Further, since $R$-ideals are assumed to be s.g. ideals, $e_{o}$ is in $I(\mathfrak{H})$. Hence $I(\mathfrak{A})$ is an $R$-ideal.

Assume that (19) holds, that $F \subset S$ and $f, g \in S$ are as in (20). For any $h \in S$, the set $\mathfrak{F}(h)=\{M \in \mathfrak{S}: h \in M\}$ is closed in the dual Stone paratopology. Hence the set $\mathfrak{A}=\bigcap\{\mathfrak{F}(f): f \in F\}$ is closed. Therefore $I(\mathfrak{H})=\bigcap\{M: M \in \mathfrak{A}\}$ is an $R$-ideal. Now $f$ and $g$ are elements of $I(\mathfrak{A})$ since $M \in \mathfrak{A}$ implies $M \cap F=\varnothing$, while both $\{f\} \cup F$ and $\{g\} \cup F$ are covering sets. Hence there is $e \in I(\mathfrak{H})$ such that $f R e$ and $g R e$. The set $\{e\} \cup F$ is covering; for if $M \in \subseteq$ and $M \cap F=\varnothing$ then $I(\mathfrak{U}) \subset M$ so that $e \in M$.

Next we show that if (20) holds and $A$ is a maximal $f$-set then $S \backslash A$ is an $R$-ideal. In virtue of Theorem 3.5, the only property which requires proof is that $S \backslash A$ is $R$-directed. For $f, g \in S \backslash A$ there is a finite subset $F$ of $A$ such that the sets $\{f\} \cup F$ and $\{g\} \cup F$ are covering. $F$ is not covering since $F \subset A$. By hypothesis there is $e \in S$ such that $f R e$ and $g R e$ and the set $\{e\} \cup F$ is covering, whence $e \in S \backslash A$. Therefore $S \backslash A$ is $R$-directed.

Now suppose $S$ consists of all proper $R$-ideals, prime $R$-ideals, or primelike $R$-ideals. According to the proof of Corollary 3.6, $\backslash \backslash A$ is a proper subset of $S$ if $A$ is a maximal $f$-set $\left(c_{1}\right)$. Hence if $S \backslash A$ is also an $R$-ideal, then $S \backslash A$ belongs to $\subseteq$ by Theorem 3.5. (To see that 3.5 applies to the collection of prime $R$-ideals, recall 2.5). According to the remarks preceding Lemma 3.3, it follows that $\subseteq$ is compact. The proof of the theorem is complete.

Following Blair [4], we say that $\mathbb{S}$ admits the dual Stone topology if the dual Stone paratopology is actually a topology. Lemma 2.2 together with [4, Theorem 1.1] yield the following theorem, though we include a short direct proof.

THEOREM 3.10. The collection of proper O-ideals of a semigroup admits the dual Stone topology. 
Proof. As we observed at the beginning of this section it is sufficient to consider two families of $O$-ideals $\mathfrak{A}_{1}$ and $\mathfrak{A}_{2}$ and show that $c_{1}\left(\mathfrak{A}_{1} \cup \mathfrak{A}_{2}\right) \subset c_{1}\left(\mathfrak{A}_{1}\right)$ $\cup c_{1}\left(\mathfrak{A}_{2}\right)$. If $J_{i}=\bigcup\left\{M: M \in \mathfrak{A}_{i}\right\}$ for $i=1,2$, and if $I$ is in $c_{1}\left(\mathfrak{A}_{1} \cup \mathfrak{A}_{2}\right)$ then $I \subset J_{1} \cup J_{2}$. By Lemma 2.2, $I \subset J_{1}$ or $I \subset J_{2}$. Therefore we have $I \in c_{1}\left(\mathfrak{A}_{1}\right)$ $\cup c_{1}\left(\mathfrak{A}_{2}\right)$.

Definition 3.11. Let $R$ be a relation on $S$ such that every $R$-ideal in $S$ is an $O$-ideal in $S$. Then the $R$-ideal space of $S$ is the topological space of all proper $R$-ideals of $S$ with the dual Stone topology. The maximal $R$-ideal space of $S$, written $\mathfrak{M}(R, S)$, is the subspace of maximal $R$-ideals. When $R=O$, this latter space is written $\mathfrak{M}(S)$.

4. $R$-semirings at $h_{o}$. Our aim in this section is to define a class of semirings which is sufficiently broad to include the $R$-lattices of Shirota [15] as well as various rings of continuous functions, and to prove some ideal-structure theorems for these semirings.

For the sake of completeness, we include the definition of $\boldsymbol{R}$-lattice. We replace the symbol " $\gg$ " of [15] by " $R$ " throughout; and the statement of (v) has been slightly modified.

Definition 4.1 (Shirota [15]). A distributive lattice $L$ is an $R$-lattice if: (1) $L$ contains a zero 0 and satisfies Wallman's disjunction property, and (2) there is a relation $R$ on $S$ such that (i) $h \geqq f$ and $f R g$ imply $h R g$; (ii) $f_{1} R g_{1}$ and $f_{2} R g_{2}$ imply $f_{1} \wedge f_{2} R g_{1} \wedge g_{2}$; (iii) $f R g$ implies there exists $h \in L$ such that $f R h R g$; (iv) for all $f \neq 0$ there exist elements $g_{1}$ and $g_{2} \neq 0$ such that $g_{1} R f R g_{2}$; (v) if $T$ is the set of triples $(h, f, g)$ such that $h R f R g$ then there is a function $\phi: T \rightarrow S$ having the following properties: (a) $\phi(h, f, g) \vee f$ $=h$, (b) $\phi(h, f, g) \wedge g=0$, and (c) if $h^{\prime} R h$ and $g R g^{\prime}$, then

$$
\phi\left(h^{\prime}, g, g^{\prime}\right) R \phi(h, f, g) \text {. }
$$

It is asserted in [15] that these hold: (vi) if $f R g$ then $f \geqq g$, and (vii) if $f R g$ and $g \geqq h$ then $f R h$. As a consequence, the relation $R$ is transitive. Statement (vi) follows easily from (iv) and (v). Statement (vii) follows from (ii) and (i) if $k \in L$ can be found such that $k R h$. If $h \neq 0$, such $k$ exists by (iv). If $h=0$, then (iv), (iii), (v) and (ii) all seem to be needed to prove the existence of such $k$.

Now let $(S,+, \cdot)$ be a semiring with identity 1 and transitive relation $R$, and consider the following restrictions which may be imposed on $S$ and $R$. To see that they all hold in an $R$-lattice, interpret $a+b$ as the greatest lower bound and $a b$ as the least upper bound of $a$ and $b$ respectively. Notice that with this interpretation, an element is a lattice-theoretic zero if and only if it is a "multiplicative" identity, and the lattice relation $\geqq$ is identical with the (multiplicative) canonical order $O$.

(22) $f R g$ implies $f O g$;

(23) $f_{1} R g_{1}$ and $f_{2} R g_{2}$ imply there is a "homogeneous" polynomial $\pi$ in two variables (with coefficients in $S$ ) such that $f_{i} R \pi\left(g_{1}, g_{2}\right)$ for $i=1,2$;

(24) $f R g$ implies there exists $h \in S$ such that $f R h R g$; 
(25) $f R g$ and $h \in S$ imply $h f R g$ and $f h R g$;

(26) $f R g$ and $h R g$ imply $(f+h) R g$;

(27) for every $R$-ideal $M, 1 \in 1+M$;

(28) $f R g$ and $f+m=1$ imply there is $g^{\prime} \in S$ such that $g+g^{\prime} m=1$.

Definition 4.2. If $S$ satisfies the following variation of (v) above for an element $h_{o} \in S$, then $S$ is relatively complemented at $h_{o}$ :

(29) There exists $h_{1} \in S$ such that $h_{1} R h_{o}$, and a function $\phi$ satisfying the following conditions: Let $T$ denote the set of triples $(h, f, g)$ such that $h_{1} R h R h_{o}$ and $h R f R g$. Then $\phi$ is a mapping from $T$ into $S$ such that (a) $\phi(h, f, g) f=h$, (b) $\phi(h, f, g)+g=1$, and (c) if $h_{1} R h^{\prime} R h$ and $g R g^{\prime}$ then $\phi\left(h^{\prime}, g, g^{\prime}\right) R \phi(h, f, g)$.

Definition 4.3. A semiring $S$, with identity 1 and transitive relation $R$, which is relatively complemented at $h_{o} \in S$, and which satisfies conditions (22)-(28) is an $R$-semiring at $h_{o}$.

Evidently an $R$-lattice is an $R$-semiring at every one of its elements. To obtain another class of examples, consider a ring $S$ with identity. Let $R$ be either the canonical or strong canonical order on $(S, \cdot)$. Then $f R g$ implies $f O g$, and $h R 0$ implies $h=0$. Define $\phi(h, f, g)=1-g$. It is easy to see that $\phi$ satisfies (a), (b) and (c) of condition (29), with $h_{1}=h_{o}=0$. Further, the conditions (22)-(28) are all satisfied except possibly the denseness condition (24). Consider (26), for example, with $R=O^{2}$. If $f O^{2} g$ and $h O^{2} g$, then there exist elements $e_{1}$ and $e_{2}$ such that $f O e_{1} O g$ and $h O e_{2} O g$. It follows that $(f+h) O\left(e_{1}+e_{2}-e_{1} e_{2}\right) O g$, and hence that $(f+h) O^{2} g$. The required polynomial in (23) is the "circle" polynomial $\pi(x, y)=x+y-x y$. Condition (28) follows since $f R g$ and $f+m=1$ now imply $g+(1-g) m=1$.

In a biregular ring with identity, for example, the canonical order is dense, since for any $f$, there exists an idempotent $e_{1}$ in the center, and elements $x, y$ and $z$ such that $f=x e_{1}$ and $y f z=e_{1}$. Hence $f O e_{1}$, and if $f O e$ for some $e$, then $e_{1} O e$. The canonical order need not be dense on rings $K(X)$; however, the strong canonical order is dense on these rings (see Remark 5.4 and the results just preceding it).

Definition 4.4. A ring with identity on which the strong canonical order is dense is an R-ring.

REMARK 4.5. It is useful to make these elementary observations: (i) an $R$-ring is an $O^{2}$-semiring at 0 with $\phi(h, f, g)=1-g$; (ii) a set $I$ is an $O$-ideal if and only if it is an $O^{2}$-ideal; (iii) in the presence of (22) and (25) every $R$-ideal is an $O$-ideal and therefore is a s.g. ideal; (iv) if $S$ is an $R$-semiring at $h_{o}, h_{1}$ is as in Definition 4.2 and $h_{1} R h R h_{o}$, then $S$ is also an $R$-semiring at $h$; (v) if the canonical order on a semigroup is dense, then it coincides with the strong canonical order. In particular, a biregular ring with identity is an $R$-ring, in virtue of the comments prior to Definition 4.4.

REMARK 4.6. If the strong canonical order is dense on a semigroup $S$ then its restriction to any Silov $(O)$ semigroup $S_{o}$ is dense on $S_{o}$. For take $f, e \in S_{o}$ 
such that $f O^{2} e$. Then there are elements $h$, and $h^{\prime} \in S$ such that $f O^{2} h O^{2} h^{\prime}$ $O^{2} e$. Since $S_{o}$ is Silov $(O)$ there are elements $e_{1}, e_{2}$, and $e_{3} \in S_{o}$ and in the center of $S$ such that $f O e_{1} O h, h O e_{2} O h^{\prime}$ and $h^{\prime} O e_{3} O e$. But then $f O^{2} e_{2} O^{2} e$, so $O^{2}$ is dense on $S_{o}$. Similar arguments show that a subset $S_{o}$ is Silov $(O)$ if and only if it is Silov $\left(O^{2}\right)$, still under the assumption that $O^{2}$ is dense on $S$.

Now take $R$ to be a fixed transitive relation on $S$. We assume that $S$ is an $R$-semiring at $h_{o}$, and study the $R$-ideal structure of $S$.

Lemma 4.7. $A$ subset $I \subset S$ is an $R$-ideal if and only if $I$ is an ideal such that

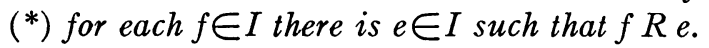

Proof. Suppose $I$ is an $R$-ideal. $I$ obviously satisfies $\left({ }^{*}\right)$; and by Remark 4.5 (iii), it is a s.g. ideal in $(S, \cdot)$. It remains to show $I+I \subset I$. Take $f_{1}, f_{2} \in I$. There is $e \in I$ such that $f_{i} e=f_{i}$ for $i=1,2$. Therefore $f_{1}+f_{2}=\left(f_{1}+f_{2}\right) e$. Hence $f_{1}+f_{2} \in I$, since $I$ is a s.g. ideal.

Conversely, suppose $I$ is an ideal satisfying $(*)$. Then $I$ is an ideal with respect to $R$, since $R$ is as strong as the canonical order. And $I$ is $R$-directed; for take $f_{1}, f_{2}, e_{1}$ and $e_{2} \in I$ such that $f_{i} R e_{i}$ for $i=1,2$. By (23), there is a polynomial $\pi$ such that $f_{i} R \pi\left(e_{1}, e_{2}\right)$ for $i=1,2$. Since $I$ is an ideal, we have $\pi\left(e_{1}, e_{2}\right) \in I$. Therefore $I$ is an $R$-ideal.

Theorem 4.8. Suppose $I_{1}$ and $I_{2}$ are $R$-ideals and let $J=I_{1} \cup I_{2}$. Then the set $I_{1} \bigvee I_{2}$ of finite sums of members of $J$ is an R-ideal.

Proof. It is immediate that $I_{1} \bigvee I_{2}$ is an ideal of $S$. By the previous lemma, it remains only to show that $I_{1} \bigvee I_{2}$ satisfies $\left({ }^{*}\right)$. It is sufficient to show that if $h=h_{1}+h_{2}$, with $h_{i} \in I_{i}$, then there is $e \in I_{1} \bigvee I_{2}$ such that $h R e$. There exist elements $e_{i} \in I_{i}$ such that $h_{i} R e_{i}$, for $i=1,2$. By (23), there is a polynomial $\pi$ such that $h_{i} R \pi\left(e_{1}, e_{2}\right)$, for $i=1,2$. By (26), we have $h R \pi\left(e_{1}, e_{2}\right)$. Since $I_{1}$ and $I_{2}$ are ideals, we have $\pi\left(e_{1}, e_{2}\right) \in I_{1} \bigvee I_{2}$, and the proof is complete.

Recall that every $R$-ideal is an $O$-ideal (by Remark 4.5 (iii)). Therefore the collection of $R$-ideals admits the dual Stone topology by 3.10. The maximal $R$-ideal space is the subspace of maximal $R$-ideals defined in 3.11.

Corollary 4.9. If $M_{1}$ and $M_{2}$ are distinct maximal $R$-ideals, then $M_{1} \bigvee M_{2}$ $=S$. Hence the maximal $R$-ideal space $\mathfrak{M}(R, S)$ of $S$ is Hausdorff.

Proof. The first result is immediate. The second follows since the sets $\mathfrak{u}(f)$ form a base for the topology of $\mathfrak{M}(R, S)$. If $M_{1} \vee M_{2}=S$, then there are elements $e_{i} \in M_{i}, i=1,2$, such that $e_{1}+e_{2}=1$. Therefore $\mathfrak{u}\left(e_{1}\right) \cap \mathfrak{u}\left(e_{2}\right)=\varnothing$.

Corollary 4.10. No proper ideal can contain distinct maximal $R$-ideals.

Proof. Obvious.

Lemma 4.11. For $e \in S$, let $L(e)=\{f \in S: f R e\}$. If $L(e)$ is non-null, then it is an $R$-ideal. If $N \subset S$ is an ideal, and if $L(N)=\{f \in S: f R$ efor some $e \in N\}$ is non-null, then $L(N)$ is an R-ideal. 
Proof. Being non-null, both $L(e)$ and $L(N)$ are s.g. ideals, by (25). By (26), $L(e)+L(e) \subset L(e) ; L(N)+L(N) \subset L(N)$ in virtue of (23). The denseness condition (24) implies that for each $f \in L(e)[L(N)]$ there is $e^{\prime} \in L(e)[L(N)]$ such that $f R e^{\prime}$. By Lemma 4.7, $\mathrm{L}(e)$ and $L(N)$ are $R$-ideals.

THEOREM 4.12. If $M$ is a maximal $R$-ideal, if $f \in M$ and $f R e$, then there is $m \in M$ such that $e+m=1$.

Proof. By definition, $f \in L(e)$, so $L(e)$ is an $R$-ideal by the previous lemma. By Theorem 4.8, $M \bigvee L(e)$ is an $R$-ideal. It properly contains $M$, since $f \notin M$, and therefore contains 1 . If $1 \in L(e)$, then by (22), $1=e$. Now by (27), there is $m \in M$ such that $e+m=1+m=1$. Otherwise we have $1 \in L(e)+M$, so for some $h R e$ and $n \in M, h+n=1$. By (28), there is $e^{\prime} \in S$ so that $e+e^{\prime} n=1$. Since $M$ is an ideal, we have $1 \in e+M$, and the lemma is proved.

Corollary 4.13. Every maximal $R$-ideal is prime-like $(R)$.

Proof. Let $M$ be a maximal $R$-ideal and suppose $f, e \in S$ such that $f \notin M$ and $f R e$. By the theorem there is $m \in M$ so that $e+m=1$. Thus for any $h \in S$ we have $h e+h m=h$. Therefore $h e \in M$ implies $h$ is in $M$, and the proof is complete.

Corollary 4.14. If $f R e$, then, in the maximal $R$-ideal space $\mathfrak{M}(R, S)$ of $S$, we have $\mathfrak{U}(f) \supset c_{1}(\mathfrak{U}(e))$.

Proof. Suppose $M_{o} \in c_{1}(\mathfrak{U}(e))$. That is, suppose

$$
M_{o} \subset \cup\{M \in \mathfrak{M}(R, S): e \in M\} .
$$

If $f \in M_{o}$, then there is $m \in M_{o}$ such that $e+m=1$. But then $m$ is in $M$ for some $M \in \mathfrak{U}(e)$. Since this is impossible we have $f \in M_{o}$.

So far, condition (29) has not been used. It is needed in the following partial converse of Corollary 4.13.

Theorem 4.15. Every prime-like $R$-ideal $M$ which contains $h_{o}$ is maximal.

Proof. Suppose $h_{1}$ is as in Definition 4.2, and that $M<M^{\prime}$, where $M^{\prime}$ is an $R$-ideal. Take $h \in S$ such that $h_{1} R h R h_{o}$ and choose $k \in M^{\prime} \backslash M$. Since $M^{\prime}$ is an $R$-ideal, there are elements $f$ and $g$ in $M^{\prime}$ such that $k R f, h R f$ and $f R g$. Hence there is an element $\phi(h, f, g)$ such that $\phi(h, f, g) f=h$ and $\phi(h, f, g)+g$ $=1$. Since $M$ is prime-like $(R)$, we have $\phi(h, f, g) \in M$, so by the second equation, $1 \in M^{\prime}$. Hence $M^{\prime}=S$, and $M$ is maximal.

The following lemma concerns the continuity of certain maps.

Lemma 4.16. Suppose that $\{M: M \in \mathfrak{M}\}$ is a collection of prime-like $R$ ideals in $\mathfrak{U}\left(h_{o}\right)$. Suppose $\mathfrak{R}$ is a collection of ideals such that $\cap\{M: M \in \mathfrak{M}\}$ $\subset \cap\{N: N \in \mathfrak{N}\}$. Let $M_{o}$ be a prime-like R-ideal in $\mathfrak{U}\left(h_{o}\right)$ and let $N_{o}$ be a proper

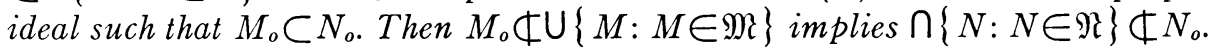

Proof. Suppose $h_{1}$ is as in Definition 4.2, and take $h$ such that $h_{1} R h R h_{o}$. There are elements $k, f, g \in M_{o} \backslash \cup\{M: M \in \mathfrak{M}\}$ such that $k R f, h R f$ and 
$f R g$. Therefore there is an element $\phi(h, f, g)$ such that $\phi(h, f, g) f=h$ and $\phi(h, f, g)+g=1$. Since each $M$ is prime-like $(R)$, the first equation implies $\phi(h, f, g)$ is in $\cap\{M: M \in \mathfrak{M}\} \subset \cap\{N: N \in \mathfrak{N}\}$, and the second implies $\phi(h, f, g) \notin N_{o}$.

TheOREM 4.17. The identity map on the collection $\mathfrak{U}\left(h_{0}\right)$ of maximal $R$-ideals containing $h_{o}$ is continuous from the Stone paratopology to the dual Stone topology.

Proof. First recall that the members of $\mathfrak{U}\left(h_{0}\right)$ are prime-like $(R)$ by 4.13 . Then apply the lemma, with $\mathfrak{M}=\mathfrak{N}$ and $M_{o}=N_{o}$.

Lemma 4.18. Let $h_{1}, h_{o}$ be as in Definition 4.2 and take $h, h^{\prime} \in S$ such that $h_{1} R h^{\prime} R h R h_{o}$. If $N$ is a proper prime-like $(R)$ ideal containing $h$, then $L(N) \equiv\{f: f R e$ for some $e \in N\}$ is a maximal $R$-ideal containing $h^{\prime}$.

Proof. The set $L(N)$ is proper, for $1 \notin N$, and as we have seen, $1 R e$ implies $1=e$. Now $L(N)$ contains $h^{\prime}$ by definition, and is an $R$-ideal by Lemma 4.11. If $M$ is a proper $R$-ideal containing $L(N)$ then, as we shall show, $M \subset N$. Therefore $M=L(N)$. For if $M \subset N$ then $L(M) \subset L(N)$; but $L(N) \subset M$ $\subset L(M)$, since $M$ is an $R$-ideal. Hence $L(N)$ is maximal.

We prove $M \subset N$ by contradiction. Suppose $k \in M \backslash N$. Take elements $q$ and $q^{\prime} \in S$ such that $h_{1} R q^{\prime} R q R h^{\prime}$. Then $q \in M$, so there are elements $f, g, g^{\prime} \in M$ such that $k R f$ and $q R f$, while $f R g R g^{\prime}$. By condition (29), there are elements $\phi\left(q^{\prime}, g, g^{\prime}\right)$ and $\phi(q, f, g)$ such that $\phi\left(q^{\prime}, g, g^{\prime}\right) R \phi(q, f, g)$, $\phi(q, f, g) f=q \in N$, and $\phi\left(q^{\prime}, g, g^{\prime}\right)+g^{\prime}=1$. Since $N$ is prime-like $(R)$, we have $\phi(q, f, g) \in N$. Therefore $\phi\left(q^{\prime}, g, g^{\prime}\right) \in L(N) \subset M$. But $g^{\prime} \in M$ and $\phi\left(q^{\prime}, g, g^{\prime}\right)+g^{\prime}$ $=1$ yield a contradiction, since $M$ is a proper ideal. It follows that $M \subset N$, and the proof is complete.

Let $\mathfrak{B}(S)$ denote the space of all proper prime-like $(R)$ ideals in $S$ with the Stone paratopology. $\mathfrak{P}(S)$ is compact by Corollary 3.6. For $h \in S$, let $\mathfrak{N}(h)$ be the collection of members of $\mathfrak{B}(S)$ which contain $h . \mathfrak{N}(h)$ is compact since it is a closed subset of $\mathfrak{P}(S)$. Suppose $h$ and $h^{\prime}$ are as in the previous lemma. Then $L$ maps $\mathfrak{R}(h)$ into the collection $\mathfrak{U}\left(h^{\prime}\right)$ of maximal $R$-ideals containing $h^{\prime}$. Regard $\mathfrak{u}\left(h^{\prime}\right)$ as a subspace of $\mathfrak{M}(R, S)$. Since the members of $\mathfrak{U}\left(h^{\prime}\right)$ are prime-like $(R)$ by 4.13 , another consequence of Lemma 4.16 is that $L$ is continuous: Replace $h_{o}$ of that lemma by $h^{\prime} . S$ is an $R$-semiring at $h^{\prime}$ by Remark 4.5 (iv). Take $\mathfrak{N} \subset \mathfrak{N}(h)$ and $N_{o} \in \mathfrak{N}(h)$. With $\mathfrak{M}=L[\mathfrak{N}]$ and $M_{o}$ $=L\left(N_{o}\right)$, the continuity of $L$ is immediate.

Now $\mathfrak{U}(h) \subset L[\mathfrak{N}(h)]$. For on the one hand, if $M \in \mathfrak{U}(h)$ then $M \in \mathfrak{N}(h)$ by 4.13. On the other hand, $M=L(M)$ since $L(M) \subset M$ for any ideal, and $L(M) \supset M$, if $M$ is an $R$-ideal. Therefore $M \in L[\mathfrak{N}(h)]$.

Finally, since $h R h_{o}$ we have $c_{1}\left(\mathfrak{U}\left(h_{o}\right)\right) \subset \mathfrak{U}(h)$ by 4.14 , where $c_{1}\left(\mathfrak{U}\left(h_{o}\right)\right)$ is the dual Stone closure of $\mathfrak{U}\left(h_{o}\right)$ in $\mathfrak{M}(R, S)$. Therefore $c_{1}\left(\mathfrak{U}\left(h_{0}\right)\right)$ is compact, being a closed subset of the compact space $L[\mathfrak{N}(h)]$. Since the sets $\mathfrak{u}(h)$ 
form a base for the maximal $R$-ideal space $\mathfrak{M}(R, S)$, we have the following generalization of a part of Shirota's Theorem 1 [15].

Theorem 4.19. If $S$ is an $R$-semiring at $h_{o}$, then $c_{1}\left(\mathfrak{U}\left(h_{o}\right)\right)$ is compact in the dual Stone topology. If $h_{o}$ is in every maximal $R$-ideal then $\mathfrak{M}(R, S)$ is compact. If $S$ is an $R$-semiring at every $h \in S$ then $\mathfrak{M}(R, S)$ is locally compact.

REMARK 4.20. Our proof of this theorem yields a different proof of the local compactness of the space of maximal open ideals in an $R$-lattice from that in [15]. This difference was not made necessary by the greater generality of semirings. Shirota's proof applies here with little change. On the other hand, a proof which might generalize to semigroups still seems to be missing.

Recall that one hypothesis of Theorem 3.9 was that $R$ satisfy the condition

(30) $f R g$ and $f R h$ imply $f R g h$.

For the remaining results, we assume

( $\alpha$ ) $S$ has a zero 0 , and further, $S$ is an $R$-semiring at 0 in which condition (30) holds.

REMARK 4.21. We shall need these consequences of $(\alpha)$ :

(i) $0 R 0$.

For by (29), there exists $h_{1}$ such that $h_{1} R 0$. By (22), $0 h_{1}=h_{1}$, so $h_{1}=0$.

(ii) $\{0\}$ is a proper $R$-ideal, so $\mathfrak{M}(R, S)$ is not empty.

The first statement follows from (i), and the second from Zorn's lemma.

(iii) $1 R 1$.

Since $0 R 0$, (29) implies that there is an element $\theta=\phi(0,0,0)$ such that $\theta+0=1$ and $\theta R \theta$, since $0 R 0$. By (23), there is a polynomial $\pi$ such that $\theta R \pi(0, \theta)$ and $0 R \pi(0, \theta)$. By $(26),(\theta+0) R \pi(0, \theta)$. Thus $1 R \pi(0, \theta)$. This implies $\pi(0, \theta)=1$, so $1 R 1$.

(iv) $h=h+0$ for every $h \in S$.

Since $1 R 1$, we have $0 R 1$, by (25). Thus there is an element $g=\phi(0,1,1)$ such that $g 1=0$ and $g+1=1$. Therefore $1=1+0$. Hence for every $h \in S$, we have $h=h+0$.

REMARK 4.22. (i) It is asserted in [15] that, in an $R$-lattice $L$, if $f_{1} R g_{1}$ and $f_{2} R g_{2}$ then $f_{1} \bigvee f_{2} R g_{1} \bigvee g_{2}\left({ }^{2}\right)$. Thus (30) holds in an $R$-lattice. Hence if

(2) Shirota has sent us a proof of this result, which we now sketch. It is sufficient to consider the case where $f_{1}$ and $f_{2}$ are equal to some $f$. Let $j, k, h, h_{i}$ and $q_{i}, i=1,2$, be elements such that $j R k R h R h_{1} R h_{2} R f$, and $f R q_{i} R g_{i}$. There are elements $\phi\left(h_{2}, f, q_{i}\right)$ and $\phi\left(h_{1}, q_{i}, g_{i}\right)$ for $i=1,2$, such that $\phi\left(h_{1}, q_{i}, g_{1}\right) R \phi\left(h_{2}, f, q_{i}\right)$. Let $a=\phi\left(h_{2}, f, q_{1}\right) \wedge \phi\left(h_{2}, f, q_{\dot{2}}\right)$ and let $b=\phi\left(h_{1}, q_{1}, g_{1}\right)$ $\wedge \phi\left(h_{1}, q_{2}, g_{2}\right)$. Take $a_{1}$ such that $b R a_{1} R a$. There are elements $\phi\left(h, b, a_{1}\right)$ and $\phi\left(k, a_{1}, a\right)$ such that $\phi\left(k, a_{1}, a\right) R \phi\left(h, b, a_{1}\right)$. We have only to prove that $\phi\left(h, b, a_{1}\right) \wedge f \geqq g_{1} \bigvee g_{2}$ and $f \geqq \phi\left(k, a_{1}, a\right)$ $\wedge h_{2}$, for then $f \geqq \phi\left(k, a_{1}, a\right) \wedge h_{2} R \phi\left(h, b, a_{1}\right) \wedge f \geqq g_{1} \vee g_{2}$. We have $b \wedge g_{1}=0$ and $b \wedge g_{2}=0$, so $b \wedge\left(g_{1} \bigvee g_{2}\right)=0$. Furthermore $\phi\left(h, b, a_{1}\right) \bigvee b=h \geqq g_{1} \bigvee g_{2}$, so $\phi\left(h, b, a_{1}\right) \geqq g_{1} \bigvee g_{2}$, and $f \geqq g_{1} \bigvee g_{2}$, which proves the first statement. To prove the second, note that $h_{2}=\phi\left(h_{2}, f, q_{1}\right) \bigvee f$ and $h_{2}=\phi\left(h_{2}, f, q_{2}\right) \bigvee f$ imply $f \bigvee a=f \bigvee\left(\phi\left(h_{2}, f, q_{1}\right) \wedge \phi\left(h_{2}, f, q_{2}\right)\right)=h_{2} \bigvee h_{2}=h_{2}$. Since $\phi\left(j, b, a_{1}\right) \wedge a_{1}=0$, we have $\phi\left(j, b, a_{1}\right) \wedge f \wedge h_{2}=\phi\left(j, b, a_{1}\right) \wedge f=\phi\left(j, b, a_{1}\right) \wedge\left(f \vee a_{1}\right) \geqq \phi\left(j, b, a_{1}\right) \wedge(f \vee a)=\phi\left(k, b, a_{1}\right)$ $\wedge h_{2}$. Therefore $f \geqq \phi\left(j, b, a_{1}\right) \wedge h_{2}$, whence $f \geqq \phi\left(k, a_{1}, a\right) \wedge h_{2}$. 
$L$ has an identity, (i.e. a zero for $(L, V)$ ), then $(\alpha)$ holds. (ii) The hypothesis $(\alpha)$ obviously holds in an $R$-ring, with $R$ in (30) and $(\alpha)$ replaced by $O^{2}$.

Lemma 4.23. Assume ( $\alpha$ ) holds. If $f \neq 0$ and $f R$, then there is a maximal $R$-ideal $M$ such that $e \notin M$.

Proof. The set $A(f)=\{h \in C(S): h f=0\}$ is an ideal. Let $I=L(A(f))$. Since $0 R 0, I$ is not empty. Therefore $I$ is an $R$-ideal by Lemma 4.11. Since $1 \notin A(f)$ we have $1 \in I$, so there is a maximal $R$-ideal $M$ containing $I$. Now there are elements $e^{\prime}$ and $e^{\prime \prime} \in C(S)$ such that $f R e^{\prime \prime} R e^{\prime} R e$. By (25), $0 f R e^{\prime \prime}$, so $0 R e^{\prime \prime}$. Since $S$ is an $R$-semiring at 0 , and $h R 0$ implies $h=0$, there are elements $\phi\left(0, e^{\prime \prime}, e^{\prime}\right)$ and $\phi\left(0, e^{\prime}, e\right)$ such that $\phi\left(0, e^{\prime}, e\right) R \phi\left(0, e^{\prime \prime}, e^{\prime}\right), \phi\left(0, e^{\prime \prime}, e^{\prime}\right) e^{\prime \prime}$ $=0$ and $\phi\left(0, e^{\prime}, e\right)+e=1$. Since $f e^{\prime \prime}=f$, we have $\phi\left(0, e^{\prime \prime}, e^{\prime}\right) \in A(f)$. Hence $\phi\left(0, e^{\prime}, e\right) \in I \subset M$. Clearly $e$ is not in $M$.

Theorem 4.24. Assume ( $\alpha$ ) holds. Then the intersection of the collection of all maximal $R$-ideals of $S$ is zero.

Proof. According to Theorem 4.19, the maximal $R$-ideal space $\mathfrak{M}(R, S)$ of $S$ is compact. Now $\mathfrak{M}(R, S)$ is not empty, by 4.21 (ii). Therefore by Theorem 3.9, $I=\bigcap\{M: M \in \mathfrak{M}(R, S)\}$ is an $R$-ideal in $S$. If $I$ contains $f \neq 0$ then it also contains $e$ such that $f R e$. But this is impossible by the lemma.

Theorem 4.25. Assume ( $\alpha$ ) holds. If $J$ is an ideal, then

$$
J=\bigcap\{M+J: M \in \mathfrak{M}(R, S)\} .
$$

Proof. It is immediate from 4.21 (iv) that $J \subset \cap\{M+J: M \in \mathfrak{M}(R, S)\}$.

Suppose $f \in \cap\{M+J: M \in \mathfrak{M}(R, S)\}$. If $M \in \mathfrak{M}(R, S)$, then there is $m \in M$ and $k \in J$ such that $f=m+k$. Take $e, e^{\prime} \in M$ such that $m R e^{\prime} R e$. By (25), $0 R e^{\prime}$, so there is an element $\phi\left(0, e^{\prime}, e\right)$ such that $\phi\left(0, e^{\prime}, e\right)+e=1$. Since $f=m+k$ and $m R e$, we have $f e=m+k e$. Therefore $f e+k \phi\left(0, e^{\prime}, e\right)$ $=m+k \phi\left(0, \epsilon^{\prime}, e\right)+k e=m+k=f$. Since $J$ is an ideal, we have $f \in f e+J$. Thus the collection $\{\mathfrak{u}(e): f \in f e+J\}$, as $e$ ranges over $S$, is an (open) cover of $\mathfrak{M}(R, S)$.

Since $\mathfrak{M}(R, S)$ is compact, there is a finite set $e_{1}, e_{2}, \cdots, e_{n} \in S$ such that every $M \in \mathfrak{M}(R, S)$ contains some $e_{i}$. There are corresponding elements $k_{1}, k_{2}, \cdots, k_{n} \in J$ satisfying the $n$ equations

(31) $f=f e_{i}+k_{i}$.

An induction shows $f \in J$. In case $n=1$, then $e_{1}$, being in every $M \in \mathfrak{M}(R, S)$, is zero, so $f$ is in $J$. In order to reduce $n$ to $n-1$ such equations, multiply both sides of $f=f e_{n}+k_{n}$ by $e_{n-1}$. Substitute the expression obtained for $f e_{n-1}$ in the $(n-1)$ st equation. The new equation, combined with the remaining $n-2$ equations, yields $n-1$ equations of the form (31).

Corollary 4.26. Assume ( $\alpha$ ) holds. If $J$ is an R-ideal, then

$$
J=\cap\{M: M \in \mathfrak{M}(R, S) \text { and } M \supset J\} .
$$


Proof. Let $J$ be an $R$-ideal. In virtue of the theorem, we can write $J$ $=I^{\prime} \cap I^{\prime \prime}$, where $I^{\prime}=\bigcap\{M+J: M \in \mathfrak{M}(R, S)$ and $M \supset J\}$ and

$$
I^{\prime \prime}=\bigcup\{M+J: M \in \mathfrak{M}(R, S) \text { and } M D J\} .
$$

If $M$ is an $R$-ideal then $M+J$ is an $R$-ideal by 4.21 (iv) and 4.8. If $M D J$ then $M+J$ properly contains $M$, so, when $M$ is maximal, $M+J=S$. Therefore $I^{\prime \prime}=S$. On the other hand, if $M \supset J$, then $M+J=M$, and the proof is complete.

5. Applications. In this section, $X$ will denote an arbitrary topological space unless mention is made to the contrary. We show first that $K(X)$ satisfies a condition which is stronger than that it be an $R$-ring.

Lenma 5.1. Let $S(X)$ denote any one of the multiplicative semigroups $K(X)$, $K^{*}(X), R(X)$ or $R^{*}(X)$. If $e, f \in S(X)$ and $f e=f$, then there are elements $e_{1}, h \in S(X)$ such that (i) $f e_{1}=f$ and (ii) $e_{1}(h e)=e_{1}$.

Proof. Suppose first that $e$ is real-valued (but $f$ is arbitrary). We show that $e_{1}$ and $h$ can actually be found in $R^{*}(X)$. Take a real number $\lambda, 0<\lambda<1$, and a bounded continuous real-valued function $\chi$ defined for all real $t$, such that $\chi(1)=1$, and if $t \leqq \lambda$ then $\chi(t)=0$. If we set $e_{1}=\chi(e)$ and $h=1 / e \bigvee \lambda$, then both $e_{1}$ and $h$ belong to $R^{*}(X)$, and $f e_{1}=f$ while $e_{1}(h e)=e_{1}$. Now for arbitrary $e$, apply the previous argument to $e \bar{e}$, where $\bar{e}$ is the complex conjugate of $e$. Then there are bounded functions $e_{1}$ and $h^{\prime}$ such that $f e_{1}=f$ and $e_{1}\left(h^{\prime} e \bar{e}\right)=e_{1}$. Hence (ii) holds with $h=h^{\prime} \bar{e}$, so the proof is complete.

Lemma 5.2. Let $S$ be a semigroup which satisfies the condition $\left({ }^{*}\right) f O e$ implies there are elements $e_{1}$ and $h \in C(S)$ such that (i) $f e_{1}=f$ and (ii) $e_{1}(h e)=e_{1}$. Then the strong canonical order $O^{2}$ on $S$ is dense.

Proof. Assume that $f O^{2} e$; that is, for some $e^{\prime} \in C(S)$, we have $f e^{\prime}=f$ and $e^{\prime} e=e^{\prime}$. Then there are elements $h_{1}, h_{2}, e_{1}$ and $e_{2} \in C(S)$ satisfying (i) $f e_{1}=f$, (ii) $e_{1}\left(h_{1} e^{\prime}\right)=e_{1}$, (i) ${ }^{\prime} f e_{2}=f$ and (ii) $e_{2}\left(h_{2} e_{1}\right)=e_{2}$. It follows from (i) ${ }^{\prime}$ and (ii)' that $f O^{2}\left(h_{2} e_{1}\right)$. On the other hand, $\left(h_{2} e_{1}\right)\left(h_{1} e^{\prime}\right)=h_{2} e_{1}$ by (ii), and since $e^{\prime} e=e^{\prime}$, we have $\left(h_{1} e^{\prime}\right) e=h_{1} e^{\prime}$. Therefore $\left(h_{2} e_{1}\right) O^{2} e$ and the proof is complete.

Hence from 5.1, we have the

Theorem 5.3. The rings $K(X), K^{*}(X), R(X)$ and $R^{*}(X)$ satisfy condition (**) and hence are R-rings.

REMARK 5.4. The canonical order on $K(X)$ is not dense in general. For $e \in K(X)$, let $E(e)=\{x \in X: e(x)=1\}$. Then $f e^{\prime}=f$ and $e^{\prime} e=e^{\prime}$ imply $N(f)^{-} \subset E\left(e^{\prime}\right) \subset N\left(e^{\prime}\right) \subset E(e)$. However it can happen that $f e=f, N(f)^{-}=E(e)$ and $E(e)$ is not open. Hence no $e^{\prime}$ such that $f O e^{\prime} O e$ can be found.

REMARK 5.5. The condition $\left({ }^{* *}\right)$ is genuinely stronger than denseness of the strong canonical order. For let $X$ be the closed unit interval and let $S$ be the ring consisting of those members of $K(X)$ each of which agrees with 
some polynomial on an open interval of the form $(1 / 2,1 / 2+\epsilon)$. It is not difficult to show that $S$ is a Silov $(O)$ semi-group of $K(X)$ which does not satisfy $\left({ }^{* *}\right)$. By Remark 4.6, the strong canonical order on $S$ is dense.

In any ring $S$, the $O$-ideals coincide with the $O^{2}$-ideals, by 4.5 (ii). If $S$ is an $R$-ring, then by 4.13 and 4.15 , the prime-like $O^{2}$-ideals and maximal $O^{2}$-ideals coincide. The comments in the paragraph just preceding Theorem 3.9 show that every prime-like $O$-ideal is maximal. However there seems to be no reason in general to think that prime-like $O^{2}$-ideals are prime-like $O$ ideals. In this connection we have the

THEOREM 5.6. In a semigroup satisfying condition (**) every prime-like $\left(O^{2}\right)$ ideal is a prime-like $(O)$ ideal.

Proof. Let $P$ be a prime-like $\left(O^{2}\right)$ ideal and suppose $f, e$ and $k \in S$ are elements such that $f \in P, f O e$ and $k e \in P$. By $\left({ }^{* *}\right)$, there are elements $h$ and $e_{1} \in C(S)$ such that (i) $f e_{1}=f$ and (ii) $e_{1}(h e)=e_{1}$. Then $f O^{2}(h e)$, and since $k e \in P$, we have $k(h e) \in P$. Since $P$ is prime-like $\left(O^{2}\right), k \in P$. Hence $P$ is prime-like $(O)$.

Corollary 5.7. A subset of $K(X), K^{*}(X), R(X)$ or $R^{*}(X)$ is a maximal $O$-ideal if and only if it is a prime-like O-ideal.

REMARK 5.8. From the fact that a prime-like $O$-ideal is maximal and a prime $O$-ideal is a prime-like $O$-ideal, it is apparent, from 2.10 , that the ring of continuous functions over the closed unit interval, for example, contains no proper prime $O$-ideals.

We shall identify the (compact) maximal $O$-ideal space of $K(X)$ after the following example. Let $\$(X)$ denote the family of open subsets of $X$ regarded as a semiring with "." "U" and " $+"=" \cap "$. Thus $\{X\}$ is a zero for $\mathbb{S}(X)$ and $\{\varnothing\}$ is an identity. For $G, H \in(S(X)$, let $G R H$ mean that $H$ is completely separated from $X \backslash G$. It will be helpful to have the following statement of Theorem 4.12 in terms of notions in $\$(X)$ :

(32) Let $g$ be a maximal $R$-ideal in $\$(X)$, and let $H_{1}, H_{2} \in \circlearrowleft(X)$ satisfy $H_{1} R H_{2}$. If for each $G \in \mathcal{G}$, we have $G \cap H_{2} \neq \varnothing$, then $H_{1} \in \mathcal{G}$.

(We are indebted to the referee for pointing out the usefulness of $(32)$ in the sequel, and for observing that it clarifies the relationship of maximal $R$ ideals to ultrafilters.)

Example 5.9. $B(X)$ satisfies (22)-(30) with respect to $R$, and hence is an $R$-semiring at $\{X\}$ which satisfies the hypothesis $(\alpha)$ of $\$ 4$. Among the properties which must be checked, only (24) and (29) might require comment. If $F R G$ then there is $e \in R(X)$ with $0 \leqq e \leqq 1$ such that $e \mid G=0$ and $e \mid(X \backslash F)=1$. Let $\chi$ and $\lambda$ be as in the proof of Lemma 5.1. Recall that $e_{1}=\chi(e)$ and $h$ $=1 / e \bigvee \lambda$, so $e_{1} \mid(X \backslash F)=1$ and $e_{1} \mid Z\left(e_{1}\right)^{\circ}=0$. Hence $F R Z\left(e_{1}\right)^{\circ}$. Since $h e \mid\left(X \backslash Z\left(e_{1}\right)^{\circ}\right)=1$ and $h e \mid G=0$, we have $Z\left(e_{1}\right)^{\circ} R G$. Therefore $R$ is dense on 
(5) $(X)$ and (24) is verified. For (29), take $h_{o}$ and $h_{1}$ to be $\{X\}$ and let $\phi(X, F, G)=X \backslash G^{-}$. Then if $F R G$ we have $\phi(X, F, G) \cup F=X$, and $\phi(X, F, G)$ $\cap G=\varnothing$. Furthermore, if $F R G R G^{\prime}$, then $\phi\left(X, G, G^{\prime}\right) R \phi(X, F, G)$. Thus (29) holds.

Now corresponding to each $x \in X$ there is an $R$-ideal $\mathcal{F}$ having the property $P(x): x \in \cap\{H: H \in \mathfrak{F}\}$ (namely, $\{X\})$. Hence it can be embedded in a maximal $R$-ideal $g(x)$ with this property. By (32), if $H_{1}$ and $H_{2}$ are open sets such that $H_{1} R H_{2}$ and $x \in H_{2}$, then $H_{1} \in \mathcal{G}(x)$. For if $G \in \mathcal{G}(x)$ then $G \cap H_{2} \neq \varnothing$, since $x \in G \cap H_{2}$. As a consequence, $\mathfrak{S}(x)$ contains every $R$-ideal having $P(x)$, and hence is the only maximal $R$-ideal with this property.

Let $\gamma(X)$ be the maximal $R$-ideal space of $\$(X)$. Let $g: X \rightarrow \gamma(X)$ denote the correspondence which sends $x$ into $G(x)$. If $A \subset X$ is a subset and $x \in A^{-}$, then $\mathcal{G}(x) \subset \cup\{g(a): a \in A\}$, so $g$ is continuous. For if $G \in \mathcal{G}(x)$, then there exists $H \in \mathcal{G}(x)$ such that $G R H$. Since there is $a \in H \cap A$, we have $G \in \mathcal{G}(a)$ as above. It is as easy to see that the image of $X$ is dense in $\gamma(X)$.

The following argument shows that every member $f$ of $R^{*}(X)$ can be extended to $\gamma(X)$ as a continuous function. If $G$ is any proper $R$-ideal, then the family of (bounded) closed sets $\left\{f(G)^{-}: G \in \mathcal{G}\right\}$ has the finite intersection property. Hence there is $\lambda_{o}$ in the intersection of this family. If $g$ is maximal, then for every open set $U$ of real numbers containing $\lambda_{o}, f^{-1}(U) \in \mathcal{G}$. For there is an open set $V$ such that $\lambda_{o} \in V \subset V^{-} \subset U$. Therefore $f^{-1}(U) R f^{-1}(V)$, and for every $G \in \mathcal{G}, f^{-1}(V) \cap G \neq \varnothing$. By (32), $f^{-1}(U) \in \mathcal{G}$. It follows that $\lambda_{o}$ is the only element in $\cap\left\{f(G)^{-}: G \in \mathcal{G}\right\}$. Define $f^{*}(\mathcal{G})$ to be this $\lambda_{o}$. Then $f^{*}$ is an extension of $f$. Further, the set of $\mathcal{G}^{\prime}$ such that $f^{-1}(V) \in \mathcal{G}^{\prime}$ is a neighborhood of $\mathcal{G}$ in $\gamma(X)$. For any such $\mathcal{G}^{\prime}$, we have $f^{*}\left(\mathcal{G}^{\prime}\right) \in V^{-} \subset U$. Hence $f^{*}$ is continuous.

Thus $\gamma(X)$ is a compact Hausdorff space which contains a continuous dense image of $X$. In addition, every bounded continuous function on $g(X)$ can be extended to a continuous function on $\gamma(X)$, so that $R^{*}(X)$ is isomorphic to $R(\gamma(X))$. Thus we have another proof of Stone's result that rings of bounded continuous functions cannot be distinguished from rings of continuous functions over compact spaces [17]. Finally, it is clear that a necessary and sufficient condition for the above correspondence $g$ to be a homeomorphism is that $X$ be completely regular and Hausdorff. The necessity is immediate. For the sufficiency, note that if $G$ is an arbitrary open set and $x \in G$ then there is an open set $H$ such that $G R H$ and $x \in H$. The properties of $\gamma(X)$ in this case distinguish it as the Stone-Čech compactification $\beta(X)$ of $X$. In virtue of example 2.11 we have a different proof from that in [1] that $\beta(X)$ can be constructed from the completely regular ends in $X$.

With the aid of the following characterization of $O$-ideals in $K(X)$ in terms of $R$-ideals in $\mathfrak{S}(X)$, we shall be in a position to identify the maximal $O$-ideal space of $K(X)$. The proof of this theorem is straightforward and we omit it. 
TheOREM 5.10. Let $\sigma$ be a function on the set of R-ideals of $B(X)$ defined by $\sigma(\mathcal{G})=\{f \in K(X): Z(f) \supset G$ for some $G \in \mathcal{G}\}$, and let $\tau$ be a function on the set of O-ideals of $K(X)$ defined by $\tau(I)=\{G \in \circlearrowleft(X): G \supset Z(f)$ for some $f \in I\}$. Then $\sigma$ is a mapping onto the set of $O$-ideals of $K(X)$, and $\tau$ is its inverse.

The restriction of $\tau$ to the maximal $O$-ideal space is a one-to-one mapping from the maximal $O$-ideal space of $K(X)$ onto the maximal $R$-ideal space $\gamma(X)$ of $\mathbb{S}(X)$. Since every basic open set of maximal $O$-ideals is mapped onto a union of basic open sets in $\gamma(X)$, this mapping is a homeomorphism. In particular, when $X$ is completely regular and Hausdorff, the maximal $O$-ideal space of $K(X)$ must be homeomorphic with $\beta(X)$. In virtue of Theorems 1.7 and 1.9 we have the following generalization of Milgram's Characterization Theorem [12].

TheOREm 5.11. The Stone-Čech compactification $\beta(X)$ of a completely regular Hausdorff space $X$ is characterized by any Silov $(O)$ subset of the multiplicative semigroup on $K(X)$, where $O$ is the canonical order on $K(X)$.

REMARK 5.12. In a conversation with the author, Milgram raised some questions concerning semigroups of matrix-valued functions. In this connection note that the previous theorem remains true if $K(X)$ is replaced by the ring $S^{n}$ of $n \times n$ matrices over $K(X)$. To prove this, it is sufficient by 1.7 and 1.9 to show that some Silov $(O)$ semigroup of $S^{n}$ characterizes $\beta(X)$. But this is easy, for the center $C\left(S^{n}\right)$ is a Silov $(O)$ semigroup of $S^{n}$. On the other hand, $C\left(S^{n}\right)$ consists of the scalar matrices, and is therefore isomorphic to $K(X)$.

The positive semirings of [16] include the semiring $R^{+}(X)$ of non-negative members of $R(X)$. In this connection we have

EXAmple 5.13. The semiring $R^{+}(X)$ is an $R$-semiring which satisfies the hypothesis $(\alpha)$ of $\S 4$, where $R$ is defined as follows: let $E(X)$ consist of all $g \in R^{+}(X)$ such that $g(x) \leqq 1$ for all $x \in X$. Define $f R g$ to mean that $g \in E(X)$, and for some $e \in E(X), f O e O g$. Note that this is the same as requiring that $g \in E(X)$ and $f O^{2} g$; for if $e \in R^{+}(X)$ and $f O e O g$, then $f O(e \wedge 1) O g$, and $e \wedge 1 \in E(X)$. Conditions (22), (25), (27) and (30) obviously hold. Conditions (23) and (26) both follow from the fact that if $g_{1}$ and $g_{2}$ are in $E(X)$ then $g_{1}+g_{2}-g_{1} g_{2} \in E(X)$. If $f R g$, then $f O^{2} g$. Now the function $\chi$ which occurs in the proof of 5.1 can be chosen to be non-negative. Thus $R^{+}(X)$ satisfies condition (**) of 5.2. Therefore there is $e \in R^{+}(X)$ such that $f O^{2} e O^{2} g$. As we observed above, this yields $f O^{2}(e \wedge 1) O^{2} g$, and $f R(e \wedge 1) R g$. Hence (24) holds. If $g \in E(X)$, then $1-g \in R^{+}(X)$. Therefore, in (28), we can take $g^{\prime}=1-g$. For (29), take $h_{1}=0=h_{o}$ and define $\phi(0, f, g)=1-g$. Suppose $f R g R g^{\prime}$. Then $g \in E(X)$, so $\left(1-g^{\prime}\right) R(1-g)$, since $1-g$ is actually in $E(X)$. Hence (29) holds.

For a final application, let $S$ be a biregular ring with identity. As we observed in $4.5(\mathrm{v}), S$ is an $R$-ring in which $O^{2}=O$. By Example 2.12, every 
ideal in $S$ is an $O$-ideal. Therefore, as special cases of $4.9,4.19,4.13,4.15$ and 4.26 , with $R=O^{2}=O$, we have the following results (the final conclusion is a part of Corollary 3 of [2]).

THEOREM 5.14. If $S$ is a biregular ring with identity, then (i) the collection of maximal ideals in $S$ forms a compact Hausdorff space in the dual Stone topology, (ii) a proper ideal is maximal if and only if it is a prime-like $(O)$ ideal, and (iii) every ideal is the intersection of all the maximal ideals containing it.

\section{REFERENCES}

1. P. S. Alexandrov, Bikompakte Erweiterungen topologischer Räume, Rec. Math. (Mat. Sbornik) N.S. vol. 5(47) (1939) pp. 403-423.

2. R. F. Arens and I. Kaplansky, Topological representation of algebras, Trans. Amer. Math. Soc. vol. 63 (1948) pp. 457-581.

3. G. Birkhoff, Lattice theory, rev. ed. Amer. Math. Soc. Colloquium Publications, vol. 25, New York, 1948. 280 .

4. R. L. Blair, Stone's topology for a binary relation, Duke Math. J. vol. 22 (1955) pp. 271-

5. P. Civin and B. Yood, Ideals in multiplicative semigroups of continuous functions, Duke Math. J. vol. 23 (1956) pp. 325-334.

6. L. Gillman and M. Henriksen, Concerning rings of continuous functions, Trans. Amer. Math. Soc. vol. 77 (1954) pp. 340-362.

7. M. Henriksen, On the equivalence of the ring, lattice and semigroup of continuous functions, Proc. Amer. Math. Soc. vol. 7 (1956) pp. 959-960.

8. E. Hewitt, Rings of real-valued continuous functions, I, Trans. Amer. Math. Soc. vol. 64 (1948) pp. 45-99.

9. N. Jacobson, $A$ topology for the set of primitive ideals in an arbitrary ring, Proc. Nat. Acad. Sci. U.S.A. vol. 31 (1945) pp. 332-338.

10. I. Kaplansky, Lattices of continuous functions, Bull. Amer. Math. Soc. vol. 53 (1947) pp. 617-623.

11. V. L. Klee, Convex sets in linear spaces, Duke Math. J. vol. 18 (1951) pp. 443-466.

12. A. N. Milgram, Multiplicative semigroups of continuous functions, Duke Math. J. vol. 16 (1949) pp. 377-383. 713.

13. J. von Neumann, On regular rings, Proc. Nat. Acad. Sci. U.S.A. vol. 22 (1936) pp. 707-

14. G. Nöebling, Grundlagen der Analytischen Topologie, Springer-Verlag, Berlin, 1954.

15. T. Shirota, A generalization of a theorem of I. Kaplansky, Osaka Math. J. vol. 4 (1952) pp. 121-132.

16. W. Słowikowski and W. Zawadowski, A generalization of maximal ideals method of Stone and Gelfand, Fund. Math. vol. 42 (1955) pp. 215-231.

17. M. H. Stone, Applications of the theory of Boolean rings to general topology, Trans. Amer. Math. Soc. vol. 41 (1937) pp. 375-481.

18. H. Wallman, Lattices and topological spaces, Ann. of Math. vol. 39 (1938) pp. 112126.

Tulane University of Louisiana, New Orleans, La.

UNIVERSITY OF KENTUCKY, LEXINGTON, KY. 